\title{
Determination of wave intensity in flexible tubes using measured diameter and velocity
}

\author{
Feng $\mathrm{J}$ and Khir AW
}

\begin{abstract}
Wave intensity (WI) is a hemodynamics index, which is the product of changes in pressure and velocity across the wave-front. Wave Intensity Analysis, which is a time domain technique allows for the separation of running waves into their forward and backward directions and traditionally uses the measured pressure and velocity waveforms. However, due to the possible difficulty in obtaining reliable pressure waveforms non-invasively, investigating the use of wall displacement instead of pressure signals in calculating WI may have clinical merits.

In this paper, we developed an algorithm in which we use the measured diameter of flexible tube's wall and flow velocity to separate the velocity waveform into its forward and backward directions. The new algorithm is also used to separate wave intensity into its forward and backward directions.

In vitro experiments were carried out in two sized flexible tubes, $12 \mathrm{~mm}$ and $16 \mathrm{~mm}$ in diameters, each is of $2 \mathrm{~m}$ in length. Pressure, velocity and diameter were taken at three measuring sites. A semi-sinusoidal wave was generated using a piston pump, which ejected 40cc water into each tube.

The results show that separated wave intensity into the forward and backward directions of the new algorithm using the measured diameter and velocity are almost identical in shape to those traditionally using the measured pressure and velocity. We conclude that the new algorithm presented in this work, could have clinical advantages since the required information can be obtained non-invasively.
\end{abstract}

\section{INTRODUCTION}

$\mathrm{W}$ AVE intensity (WI) is a novel hemodynamic index, which is defined as the flux of energy carried by the wave per cross sectional area. WI is determined as the product of changes in pressure and velocity across the wavefronts. Wave intensity analysis (WIA) was introduced by Parker and Jones [1], and has been used since in the study of blood flow in aorta [2], coronary and radial arteries.

The investigation of using the diameter signals in place of the pressure signals is motivated by potential benefit of acquiring the data non-invasively. Sugawara et al. has studied the relationship between the pressure and diameter of the carotid artery in humans and found the carotid arterial pressure-diameter relationship could be regarded as linear and the vessel diameter changes were converted to pressure waveforms by scaling its peak and minimum values to

Manuscript received March 28, 2007. This work was supported in the school of engineering and design of Brunel University.

J. Feng is with the school of engineering and design in Brunel University, UB8 3PH, UK. (phone: 44-18952-67080; fax: (44) 01895 274608; e-mail: jiling.feng@ brunel.ac.uk).

A.W. Khir is with the School of Engineering and Design and Brunel Institute of Bioengineering of Brunel University, Uxbridge, UB8 3PH, UK. (e-mail: ashraf.khir@brunel.ac.uk). systolic and diastolic brachial blood pressures [3]. Hartley et al. had developed non-invasive Doppler method for arterial motion in mice and concluded that the diameter signals can replace pressure signals for calculating the augmentation index [4]. However, Van Bortel et al. pointed out that the pulse pressure is not constant throughout the arterial tree and use of pulse pressure at one arterial site as surrogate for pulse pressure at another arterial site may be erroneous [5]. O'Brien has also studied the relationship between pressure and diameter in the pulmonary artery of dogs [6].

In this work we consider a new algorithm, using diameter and velocity to determine WI. The new algorithm is also used to separate flow waveforms into forward and backward directions. A comparison between the WI results of the new algorithm and those obtained using pressure and velocity is also presented.

\section{THEORETICAL CONSIDERATION}

\section{A. Separation of waveforms using measured pressure and velocity}

WIA is based on solution of the method of characteristics to the 1-D mass and momentum equations of flow in a uniform elastic tube. The water hammer equation is

$d P_{ \pm}= \pm \rho c d U_{ \pm}$

where, $d P$ and $d U$ are the changes in pressure and velocity, $\rho$ is density of fluid, " \pm " indicates the forward and backward directions and $c$ is wave speed.

Using WIA, the measured pressure and velocity differences can be separated into the forward and backward directions

$$
\begin{aligned}
& d P_{ \pm}=1 / 2(d P \pm \rho c d U) \\
& d U_{ \pm}=1 / 2(d U \pm 1 / \rho c d P)
\end{aligned}
$$

The forward and backward pressure and velocity waveforms can be calculated

$$
\begin{aligned}
& P_{+}=P_{0}+\sum_{0}^{T} d P_{+}, P_{-}=\sum_{0}^{T} d P_{-} \\
& U_{ \pm}=\sum_{0}^{T} d U_{ \pm}
\end{aligned}
$$

Where, $P_{ \pm}$represents forward and backward pressure, $P_{0}$ is the initial pressure, $U_{ \pm}$is the forward and backward velocity and $T$ is the time of one cycle. Similarly, wave intensity can be separated into the forward and backward directions. 
$d I_{ \pm}= \pm \frac{1}{4 \rho c}(d P \pm \rho c d U)^{2}$

Separation of waveforms using measured diameter and velocity

It is well established that wave speed is a function of distensibility of tube wall

$c^{2}=\frac{A d P}{\rho d A}$

where, $A$ and $d A$ are the initial cross-sectional area and its change respectively. Re-arrange (5) gives

$d P=\rho c^{2} \frac{d A}{A}$

If $A=\frac{\pi}{4} D^{2}$ and $d A=\frac{\pi}{2} D d D$, where, $D$ and $d D$ are the diameter of tube and its change respectively, we can write $\frac{d A}{A}=\frac{2 d D}{D}$

Substitute (7) into (6) gives,

$d P=\rho c^{2} \frac{2 d D}{D}$

Because the change in pressure $\mathrm{dP}$ can be considered as the linear summation of the change in the pressure in the forward and backward direction, $\boldsymbol{d P}=\boldsymbol{d} \boldsymbol{P}_{+}+\boldsymbol{d} \boldsymbol{P}_{-}$, it is reasonable to assume that change in diameter, $\mathrm{dD}$, can also be considered as the linear summation of diameter changes due to changes in the forward and backward pressure changes, $\boldsymbol{d D}=\boldsymbol{d} \boldsymbol{D}_{+}+\boldsymbol{d} \boldsymbol{D}_{-}$

Substitute (8) into (1)

$d D_{ \pm}= \pm \frac{D}{2 c} d U_{ \pm} \quad$ or $d U_{ \pm}= \pm \frac{2 c}{D} d D_{ \pm}$

If we assume the change in the velocity waveform, $\mathrm{dU}$, is the result of the algebraic summation of the changes in the forward and backward directions, we can write

$d U=d U_{+}+d U_{-}$

Substitution $d U_{ \pm}$from equation (10) into (11) gives

$d U=\frac{2 c}{D} d D_{+}+\left(-\frac{2 c}{D} d D_{-}\right)$

The change in diameter resulting from the change in pressure in the forward and backward directions can be obtained by multiplying (9) by $(2 \boldsymbol{c} / \boldsymbol{D})$, adding and subtracting (12), and rearrange

$d D_{ \pm}=\frac{1}{2}\left(d D \pm \frac{D}{2 c} d U\right)$

To determine the change in velocity in the forward and backward directions we substitute (10) into (9) $d D=\frac{D}{2 c} d U_{+}+\left(-\frac{D}{2 c} d U_{-}\right)$

The velocity differences in the forward and backward directions can be similarly determined by multiplying (11) by $(\boldsymbol{D} / 2 \boldsymbol{c})$, adding (14), and multiplying (11) by $(\boldsymbol{D} / 2 \boldsymbol{c})$, subtracting (14) and rearrange

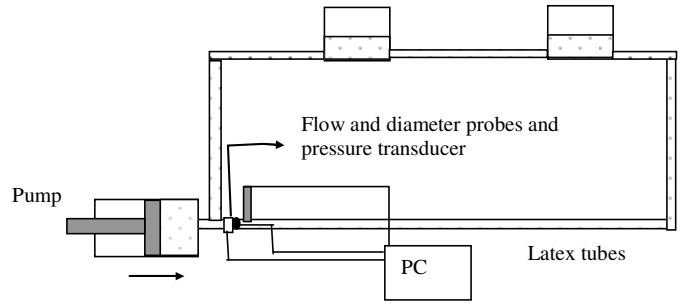

Fig.1 Experiment setup, latex tubes connect with the pump and two reservoirs, respectively. Flow, diameter probes and tipped catheter pressure transducer are used measured flow, diameter and pressure simultaneously at same position. A PC was used to acquire all data. Arrow indicates the direction of piston.

$d U_{+}=\frac{1}{2}\left(d U+\frac{2 c}{D} d D\right)$

The forward and backward diameter and velocity can be obtained by summation of changes in diameter and velocity in the forward and backward directions.

$D_{+}=\sum_{1}^{n} d D_{+}+D(0)$

$$
\begin{aligned}
& U_{+}=\sum_{1}^{n} d U_{+} \\
& U_{-}=\sum_{1}^{n} d U_{-}
\end{aligned}
$$

$D_{-}=\sum_{1}^{n} d D_{-}$

Wave intensity can then be determined by multiplying the respective direction of the change in diameter and velocity diameter changes; $d D_{+} d U_{+}$is wave intensity in the forward direction and $d D_{-} d U_{-}$is wave intensity in the backward direction.

$$
\begin{aligned}
& d D_{+} d U_{+}=\frac{1}{4(D / 2 c)}\left(d D+\frac{D}{2 c} d U\right)^{2} \\
& d D_{-} d U_{-}=-\frac{1}{4(D / 2 c)}\left(d D-\frac{D}{2 c} d U\right)^{2}
\end{aligned}
$$

\section{METHODS}

The experiment setup was shown in Fig.1, the system of which is composed of a piston pump, tank with water inside, two reservoirs, and latex tubes (two sizes: $12 \mathrm{~mm}$ and $16 \mathrm{~mm}$ in diameter). The pump and reservoirs connect the both ends of latex tube respectively through the rigid connectors. The latex tube immerged into the water in the tank about $1 \mathrm{~cm}$ in depth. The pump generates a single positive half sinusoidal wave by pushing the piston forward. The simultaneous measurements of pressure, flow and diameter were taken at $30 \mathrm{~cm}, 90 \mathrm{~cm}$ and $150 \mathrm{~cm}$ away from inlet along the $2 \mathrm{~m}$ length tube. Pressure, flow and diameter are measured using tipped catheter pressure transducer, ultrasonic flow probe and ultrasonic paired crystals, respectively. All the data were acquired program written using Labview and sampling rates is 500. The analysis was carried out using programs written in Matlab. 

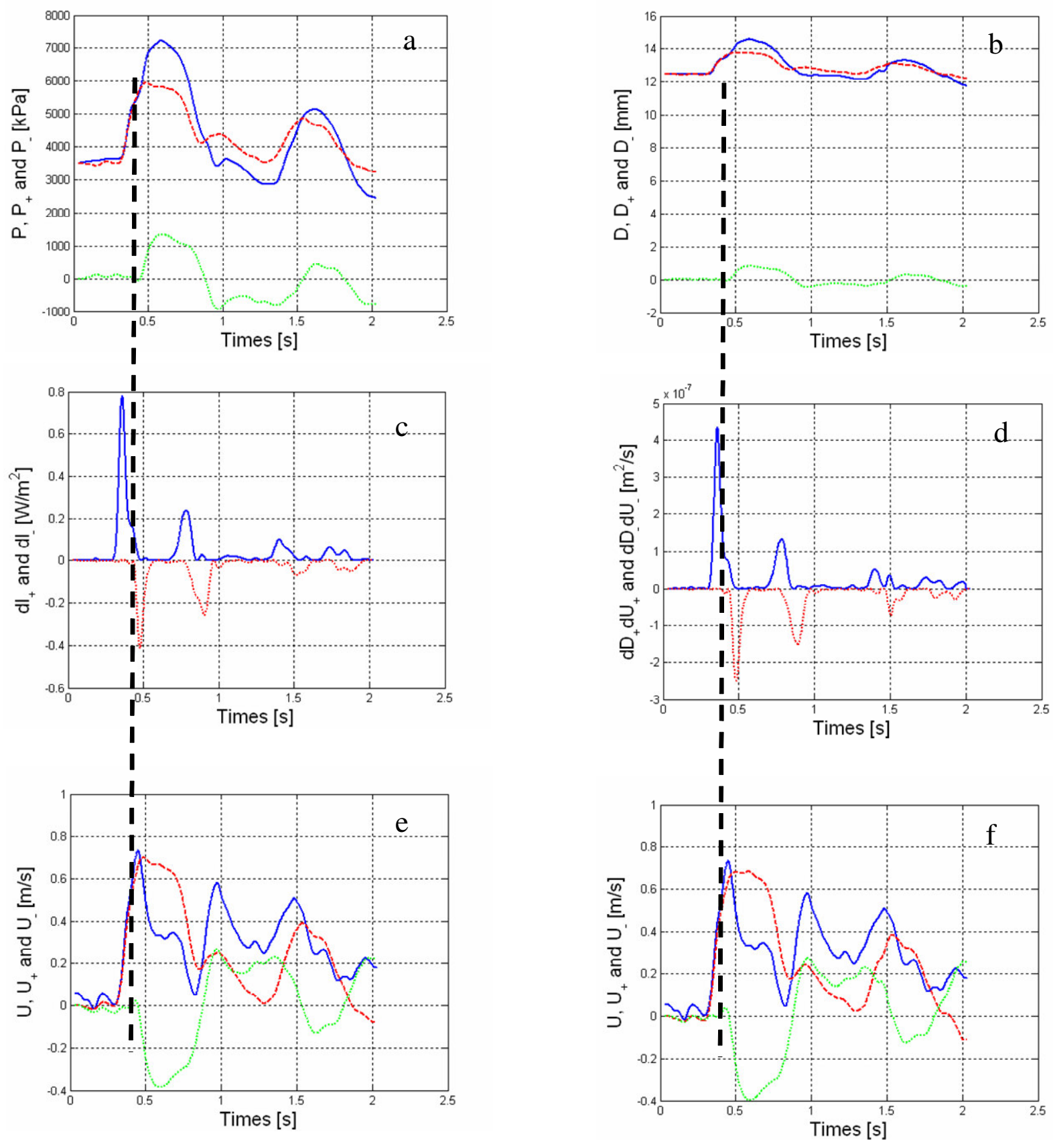

Fig. 2. The left hand side panels show the separated pressure, wave intensity and velocity waveforms using pressure and velocity of the traditional wave intensity analysis technique. Plots in the right hand side panels have the separated pressure, wave intensity and velocity waveforms using diameter and velocity of the new algorithm. The measured (solid), forward (dash) and backward (dotted) pressure, diameter and velocity are presented in a, b, e and f respectively. Forward and backward wave intensity is in red and blue respectively and presented in Fig2c and d respectively. Note the striking similarity in the waveform calculated using the new algorithm and those calculated the traditional WIA. Vertical dashed lines indicate that the arriving time of backward waveforms.

\section{RESULTS}

Fig.2 shows that separation of waveforms into forward and backward directions using measured pressure and velocity (left panels) and the measured diameter and velocity (right panels) at $150 \mathrm{~cm}$ away from inlet in the tube of $16 \mathrm{~mm}$ in diameter, respectively.

From these figures, one can see that measured pressure, $\mathrm{P}$, and its separated forward and backward pressure, $P_{ \pm}$, are very similar to the corresponding diameters, (Fig.2a and b). The reflection time determined by the backward pressure (dotted, Fig.2a) is very close to that determined by the new algorithm, (dotted, Fig.2b). Before the reflection wave arriving, the forward pressure (dashed, Fig.2a) is similar in shape to the measured pressure (solid, Fig.2a). The same is true with measured 
and forward diameter. The measured pressure and diameter increase sharply when the reflection wave arrives. Similarly to the forward and backward wave intensity determined by the pressure and velocity, the forward and backward diameter wave intensity determined by the diameter and velocity also has two peaks; first one represents the compression wave and the second one represents expansion wave. Furthermore, the forward and backward velocity determined by the measured pressure and velocity are almost identical in shape to those determined by the measured diameter and velocity.

All figures show that onset of reflection wave established using the measured pressure and velocity is very similar to that determined using diameter and velocity.

Table1 lists the reflection times at the three measurement sites $(30,90$ and $150 \mathrm{~cm})$ in the tube of $12 \mathrm{~mm}$ and $16 \mathrm{~mm}$ in diameter. In this table, we compared the reflection time established by WIA with that by diameter WIA. The results show that there is small difference between the reflection time determined by the WIA (T_pu) and by the diameter WIA (T_du). The greatest difference of reflection time amongst these two methods is $6.7 \%$ and average difference is $0.67 \%$.

\section{V.DISCUSSIONS AND CONCLUSIONS}

Determination of wave intensity using the measured diameter rather than the measured pressure can be advantageous in calculating wave intensity non-invasively. Not only reflection time determined by WI using diameter and velocity is close to that determined by WI using pressure and velocity, but the shape of the curves is very similar (Figure2c and d).

Sugawara [3] et al. found that the pressure and diameter-change waveforms of carotid arteries are very similar. Hence, they calculated the WI using the measured velocity waveform and the pressure waveforms, which is converted from the diameter change waveforms of carotid arteries [7]. In contrast, the diameter WI method developed in this paper can be used to separate the waveform into the forward and backward directions and calculate diameter WI directly from the measured diameter and velocity. The extreme similarity between the WI and diameter WI shown in Fig2 $\mathrm{c}$ and d implies that the diameter WI might be used to supply the information of the upstream and downstream of the measured arterial flow as did WI, although non-invasively.

The separation of waveform into forward and backward directions using whether the pressure or diameter required the precondition of the known wave speed, which is determined here using the PU-loop method [8]. According to the PU-loop, the wave speed is established by the measured pressure and velocity. In this case, the diameter WI method is restrained by the acquiring the local wave speed accurately only through the measured diameter and
TABLE 1THE COMPARISON OF REFLECTION TIME DETERMINED BY

THE WIA AND DIAMETER WIA

\begin{tabular}{|c|c|c|c|c|c|c|}
\hline \multirow{2}{*}{$\begin{array}{c}\begin{array}{c}\mathbf{D} \\
(\mathbf{m m})\end{array} \\
16\end{array}$} & \multirow{2}{*}{$\begin{array}{c}\begin{array}{c}\text { MS } \\
(\mathbf{c m})\end{array} \\
30\end{array}$} & \multicolumn{2}{|c|}{ T_pu (s) } & \multicolumn{2}{|c|}{ T_du (s) } & \multirow{2}{*}{$\begin{array}{l}\text { Diff } * \% \\
-2.8902\end{array}$} \\
\hline & & $d I-$ & 0.865 & $d D . d U$ & 0.89 & \\
\hline & & $P-$ & 0.878 & $D$ & 0.866 & 1.3667 \\
\hline & 90 & $d I-$ & 0.595 & $d D_{-} d U_{-}$ & 0.572 & 3.8655 \\
\hline & & $P$ & 0.594 & $D$ & 0.59 & 0.6734 \\
\hline & 150 & $d I-$ & 0.229 & $d D d U$ & 0.228 & 0.4367 \\
\hline \multirow{7}{*}{12} & & $P$ & 0.224 & $D$ & 0.226 & -0.8929 \\
\hline & 30 & $d I-$ & 0.755 & $d D . d U$ & 0.73 & 3.3113 \\
\hline & & $P$ & 0.74 & $D$ & 0.725 & 2.0270 \\
\hline & 90 & $d I-$ & 0.475 & $d D . d U$ & 0.493 & -3.7895 \\
\hline & & $P$ & 0.49 & $D-$ & 0.47 & 4.0816 \\
\hline & 150 & $d I-$ & 0.135 & $d D . d U$ & 0.144 & -6.6667 \\
\hline & & $P-$ & 0.135 & $D-$ & 0.14 & -3.7037 \\
\hline Mean & & & 0.509 & & 0.506 & 0.671 \\
\hline
\end{tabular}

Note: D represents the size of the tube; MS means the distance of measuring sites away from inlet; T_pu means the reflection time determined by the WIA; T_du means the reflection time determined by the diameter WIA; Diff*\% means the percentage of difference of $T \_p u$ and $T$ _du over the T_pu.

velocity. Therefore, a further study of establishing the wave speed only using the measured diameter and velocity will be required.

We concluded that wave intensity can be determined using diameter instead of pressure, and velocity. The new algorithm could be advantageous as it uses data that can be measured non-invasively.

\section{REFERENCES}

[1] Parker, K. H., Jones, C.J.H., "Forward and backward running waves in the arteries: analysis using the method of characteristics," Transactions of ASME: Journal of Biomechanical Engineering., vol, 112, pp.322-326, 1990

[2] A.W., Khir and K.H., Parker, "Wave intensity in the ascending aorta: effect of arterial occlusion". Journal of Biomechanics, vol 38, pp.647-655, 2005.

[3] M. Sugawara, K. Niki, H. Furuhata, S. Ohhishi, S. Suzuki, "Relationship between the pressure and diameter of the carotid artery in humans" Heart Vessels, vol 15:49-51, 2000.

[4] C. J. Harley, etc, "Non-invasive ultrasonic measurement of arterial wall motion in mice" AJP-Heart, vol. 287, pp. 1426-1432, 2004.

[5] Van Bortel LM et al. "Non-invasive assessment of local arterial pulse pressure: comparison of applanation tonometery and echotracking" J Hyperens. Vol. 19(6), pp.1037-44, Jun 2001.

[6] O'Brien A. Pulmonary hemodynamics. PhD thesis. Imperial College, London, 2003.

[7] N. Ohte, et al. "Clinical usefulness of carotid arterial wave intensity in assessing left ventricular systolic and early diastolic performance" Heart Vessels, vol. 18, pp.107-111 2003.

[8] A.W. Khir, A. O'Brien, J. Gibbs, K. H. Parker, Determination of wave speed and wave separation in the arteries. Journal of Biomechanics, vol. 34, 1145-1155 2001. 